\title{
Study on the Relationship among Parents' Cognition on China Anti-domestic Violence, Attitude of Beating Children and Frequency of Beating Children
}

\author{
Shi, Song ${ }^{1}, \mathrm{Wu}$, Hotang ${ }^{2, *}$ \\ ${ }^{1}$ Education Science College, Jilin Normal University, China \\ ${ }^{2}$ College of Education, National Kaohsiung Normal University, Taiwan
}

Copyright $\odot 2018$ by authors, all rights reserved. Authors agree that this article remains permanently open access under the terms of the Creative Commons Attribution License 4.0 International License

\begin{abstract}
This research aims at analyzing the correlation between parents' awareness of anti-domestic violence in China, attitude and frequency of beating children. According to the literature analysis, this paper sets children's parents' anti-domestic violence cognition and attitude of beating children as independent variable, and the frequency of beating children as dependent variables. It adopts self-edited Anti-family Violence Cognitive Scale and Sale of Attitude of Beating Children, investigates 2 to 6 years old children's parents $(N=957)$ in Jilin Province of China. After the hierarchical regression analysis, it shows that the anti-domestic violence cognition and attitude of beating children have the explanatory power on the frequency of beating children.
\end{abstract}

Keywords Cognition on China Anti-domestic Violence, Attitude of Beating Children, Frequency of Beating Children

\section{Introduction}

"Parents beating children" is a common daily life phenomenon in China known to all people, since the ancient time, there has been the proposal of beating children, for example, in Han Dynasty, Sima Qian (B.C 145 - B.C 90) proposed "not abandoning swapping at home"'(Zhang, 2013)[1]. In the Northern Dynasty, Yan Zhitui (A.D 530 - 591) even mentioned that "Not until the child has formed a bad habit of arrogance do his parents pay attention to subject him, even swapping him to death will be in vain, only to add his resentment day after day" (Chen, 1973)[2]. Up to now, we have usually heard about parents beating their child in social news, for example, in 2015 there is a foster mother in Nanjing who beat her child badly because of her child's problem in study, and she was in the vortex of public opinions because of this and attracted highly attention and discussion in society. Study researches in recent years have also shown that the phenomenon of beating children in family is disappearing with the development and progress of politics, economy, and culture in society, instead, it widely exists though. (Wang, Chen, \& Ma, 2007; Xiao \& Chen, 2011; He, 2014)[3, 4, 5] Quote Comenius's dictum, “It doesn't seem to stay in concealment since the ancient time", "Educators are with bitter hatred and trying to find a solution." (Lin, 2002)[6].

With regard to the phenomenon of parents beating their children, the public has been arguing about its advantages and disadvantages. Corporal punishment was associated with only one desirable behavior, namely, increased immediate compliance. Corporal punishment at the same time can also lead to the following consequences: decreased moral internalization, increased child aggression, increased child delinquent and antisocial behavior, decreased quality of relationship between parent and child, decreased child mental health, increased risk of being a victim of physical abuse(Gershoff, 2002)[7]. As early as in Rome times, Quintilian claimed that "physical punishment is disgusting", and "physical punishment should be forbidden". (Lin, 2002)[6]. In order to forbid physical punishment, China issued Anti-Domestic Violence Law of People's Republic of China(Anti-Domestic Violence Law) in 2016[8], esteeming beating family members including children as illegal.

The Anti-Domestic Violence Law (2016)[8] pointed out that "domestic violence means the behaviors between family members of beating, bounding, injuring, restricting personal freedom, and usual mental or physical prejudice in the way of abusing or frightening", and wrote it into the 12th rule that "the guardians of juveniles should do domestic education in a good manner, fulfilling the obligations of guarding and education by law and mustn't make domestic violence", although the police won't pay the public security punishment due to domestic violence is not that harmful to society, the police needs to issue "critical education or expostulating letter" to the inflictor. 
According to this law we can conclude that no matter heavy or light, as long as parents beating their children, it will be considered as domestic violence.

Followed on the discourse above, the Anti-Domestic Violence Law (2016) [8] has been implemented for more than one year, and under new social environment, if parents aware their domestic violence behavior is illegal, their frequency of beating children will must be influenced.

On the other hand, in the traditional concept of the Chinese people, "adults beating children" is reasonable (Guan, 2006) [9], namely some parents consider it not a big deal for "beating children" are because they had been treated that way in their childhood, so they think it not wrong to beat their own children now. Certainly, some parents dote on their children very much, whether their children are right or wrong, they could treat them patiently and be compliant to them. They figure that their children are still young and naive, and it would be useless to criticize or beat them (Sun, 1993)[10]. So we can see, it is parents' attitude that decide whether or not to beat children.

It has been pointed out in Gao and Ye's(2006)[11] research that parents beating children maybe comes from the purpose of correcting children's behaviors, which may be noncompliance or doing something in danger, and maybe because of parents' bad mood, or considering beating children is a vital approach of education, which comes from the traditional Chinese concept of "stick parenting may cultivate a dutiful son" and "talents are often made after stick parenting". No matter for what kind of reason, these attitudes may influence parents' frequency of beating children.

What's more, the Anti-Domestic Violence Law (2016)[8]may influence parents' attitude towards beating children, because the law would constrict people's behavior. It has been clearly stipulated in the Anti-Domestic Violence Law that our country forbids any form of domestic violence. With further understanding of this law, parents would avoid educating children by beating them as much as possible.
From the analysis above we could know that there is correlation among parents' cognition on the Anti-Domestic Violence Law, their attitude towards beating children, and their frequency of beating children, but researches in the past didn't have further study on the correlation among them. This research adopts hierarchy regression analysis to analyze relevant situation, and the hierarchy regression analysis uses the block way to put explaining variables to hierarchy regression. The order of block entering the regression equation is decided by the order of influence on dependent variable (the frequency of beating children). Firstly, demographic variables like children's age and genders, parents' education backgrounds, living region, and family structures will not be influenced by other explaining variables, so it is put in block one, and as control variable, the block two is the cognition of anti-domestic violence, while block three is the attitude towards beating children.

\section{Research Design}

\subsection{Research Sample}

This research takes parents who have kids from 2-6 years old in China's Jilin province as sample, and collect data by means of the internet questionnaire platform "So Jump". Using the channel of the Wechat group of a kindergarten in Jilin province and graduate students majoring in preschool education from three universities in Jilin Province, by means of forwarding by teachers, let parents fill out the questionnaires. The online questionnaire is in anonymity so that information of participants will be kept in secret, and in the foreword there is the introduction of the study and its function, as well as promising of keeping personal information in secret and strictly obeying academic ethics and standards.

The total of research samples is 957 . The allocation of demographic variable is in table 1 .

Table 1. Allocation of demographic variable

\begin{tabular}{|c|c|c|c|}
\hline item & gender & frequency & percentage \\
\hline \multirow{4}{*}{ Children's age } & $2 \leq$ age $<3$ years old & 66 & 6.9 \\
\hline & $3 \leq$ age $<4$ years old & 225 & 23.5 \\
\hline & $4 \leq$ age $<5$ years old & 303 & 31.7 \\
\hline & $5 \leq$ age $\leq 6$ years old & 363 & 37.9 \\
\hline \multirow[t]{2}{*}{ Children's gender } & Boy & 477 & 49.8 \\
\hline & girl & 480 & 50.2 \\
\hline \multirow[t]{6}{*}{ Parents' educational background } & Junior high & 266 & 27.8 \\
\hline & Senior high & 106 & 11.1 \\
\hline & Technical secondary & 98 & 10.2 \\
\hline & College degree & 171 & 17.9 \\
\hline & Bachelor & 262 & 27.4 \\
\hline & Master and above & 54 & 5.6 \\
\hline \multirow[t]{2}{*}{ Living region } & Urban area & 757 & 79.1 \\
\hline & Rural area & 200 & 20.9 \\
\hline
\end{tabular}




\subsection{Research Tool}

After consulting literatures in China and abroad, the researcher edited questionnaires, one is Scale of Cognition of Anti-Domestic Violence, and the other one is Scale of Attitude towards Beating Children in Likert's seven-points style (1: Totally disagree, 2: Very disagree, 3: A little disagree, 4: Not sure, 5: A little agree, 6: Very agree, 7: Totally agree). After completing the first edition, the research invited three teachers who once worked in major of preschool education in college to have examine on the first draft of scales. Afterwards, there was a try-out by comparisons of extreme groups, the exploratory factor analysis, the maximum likelihood estimation method with varimax rotation and the criterion of eigenvalue greater than 1.00, and the results of which were as follows:

\subsubsection{Scale of Cognition on Anti-Domestic Violence}

This scale based on the main content of Anti-Domestic Violence in the People's Republic of China, and the authors come up with 4 items for this scale. After the try out, results showed that the $\mathrm{CR}$ (critical ratio) value was between 4.60 and 16.62, all of which were greater than 3.0, and the $p$ was less than.000, indicating that the identification of all items was discriminating.

Next, in the factor analysis, it has adopted principal component analysis and orthogonal rotations, and the results showed that the eigenvalue is 2.28 , and the total variance is $57.06 \%$. However, the factor loading of "beating the child, if it is serious, the government departments should intervene" is in the amount of only .15, no more than .30 standard, so it has been deleted. After deletion, the results show that the eigenvalue is 2.10 , the total variance interpretation is $70.09 \%$, and the alpha coefficient is .78 .

\subsubsection{Scale of Attitude of Beating Children}

The first draft of the Scale of Attitude of Beating Children has 12 items, The process of try-out is the same with the Scale of Cognition of Anti-domestic Violence, and here's the result: the CR value is between 3.01 to 8.17 , being greater than 3 , and $p$ values are all less than .001 , indicating that all items discrimination are good.

Secondly, results of factor analysis are that three factors were named:1. The Purposiveness: (5 items), namely the purpose of parents beating children. The items are entitled like "I beat my child for letting him or her know what should do and what mustn't do", "I beat my child for his or her own good"; 2. Indirect Blasting Fuse (4 items): including "the pressure from work and life makes me beat my child", "beating my child is related to my bad mood at that time". 3. Conceptual Factor (3 items): including "no beating children, no talents", "wolf fathers and tiger mothers popular on the internet", and "beating child once every three days, he would be able to be admitted to Peking University". The total variance explained is $61.83 \%$, and the total alpha coefficient is .74.

\subsection{Data Process and Analysis}

\subsubsection{Hierarchical Regression Analysis with SPSS 22.0}

This research used SPSS 22.0 to conduct hierarchical regression analysis. It is divided into 3 models, which were adopted to thrust each variable to the equation for understanding of explanatory power. From literature analysis discussed above, this study adopted hierarchical regression analysis to explore the correlation among parents' cognition on anti-domestic violence, their attitude towards beating children and their frequency of beating children. The demographical variables are put into equation, Called Model 1, and then parents' cognition on anti-domestic violence, called model 2 , and the last one is parents' attitude towards beating children, called model 3 .

\subsubsection{Control Variables to Dummy}

Among the control variables in this study, children's gender and living region are nominal variables, so we employed dummy coding. For example, the male is dummied as 0 and female as 1 . The living region are classified as urban and rural areas, the former one is dummied as 0 , and the latter one as 1. Children's age, parents' educational background, parents' age are ordinal variables, they were thrust into the equation directly. In addition, the independent variable and the dependent variable are ratio variables, so they entered the equation with the row score.

\section{Results}

\subsection{Frequency of Beating Children}

After the research, it has been found that the frequency of beating children is: Never for 53 people (5.5\%), Rarely for 329 people $(34.4 \%)$, Sometimes for 540 people $(56.4 \%)$, Usually for 33 people (3.4\%), Always for 2 people $(0.2 \%)$. The average number is $2.58(S D=0.66)$, indicating that the frequency of beating children is between Rarely and Sometimes.

\subsection{Hierarchical Regression}

According to the result of literature analysis, it adopts the hierarchical regression and puts demographical variables, parents' cognition on anti-domestic violence, attitude towards beating children into the regression equation based on its order, and the results are as follows:

Regarding Model 1, it is mainly composed by children's age, children's gender, parents' age, parents' educational background, and living region (see Table 2). Model 1's $R^{2}$ $=.234, F=11.007$, and $p<.000$, presenting such demographic variables have significant explanatory power for the frequency of beating children. Among the five demographic variables, children's age, children's gender, 
parents' educational background, which are not statistically significant, but the parents' age $(\beta=-.118, t=$ $-3.622, p<.000)$, living region $(\beta=.125, t=3.513, p<.000)$ have significant explanatory power.

Concerning Model 2, after controlling the five demographic variables' explanatory power, we thrust dimensions of the cognition on anti-domestic violence to the equation. The model's explanatory power is $R^{2}=.305$, $F=16.195, p=.000$, showing Model 2 has explanatory power. As for $\Delta R^{2}=.038, \Delta F=39.885$, and $p=.000$, showing that after thrust each dimension of the cognition on anti-domestic violence, increment of model 2 has statistical meaning; that is, each dimension of the cognition on anti-domestic violence can increase extra $3.80 \%$ 's explanatory power. And the cognition on anti-domestic violence $\mathrm{k}(\beta=-.197, t=-6.315, p=.000)$ has the explanatory power, and three $\beta$ values are negative, signifying that the higher the cognition on anti-domestic violence, the less frequency of beating children will be.

After controlling the explanatory power of Model 1 and 2 , dimensions of the attitude towards beating children were thrust into Model 3, and obtained the explanatory power $R^{2}=.446, F=26.069$, and $p<.000$, showing Model 3 has explanatory power. As for $\Delta R^{2}=.106, \Delta F=41.660$, and $p=.000$, the increment of model 3 has statistic meaning that can contribute extra $10.60 \%$ of explanatory power.

Among the three dimensions, purposive factor $(\beta=.252$, $t=8.072, p<.000)$, indirect blasting fuse $(\beta=.180, t=5.896$, $p=.000$ ) have the significant explanatory power, $\beta$ values are all positive, showing that the more purposive factor, and indirect blasting fuse, the more frequency of beating children will be.

Table 2. Summary of hierarchical regression

\begin{tabular}{|c|c|c|c|c|c|c|c|c|c|}
\hline & \multicolumn{9}{|c|}{ Dependent variable (frequency of beating children) } \\
\hline & \multicolumn{3}{|c|}{$\begin{array}{c}\text { Model } 1 \\
\text { Control variable } \\
\text { (demographic variable) }\end{array}$} & \multicolumn{3}{|c|}{$\begin{array}{c}\text { Model } 2 \\
\text { Independent variable } \\
\text { (cognition on anti-domestic } \\
\text { violence) }\end{array}$} & \multicolumn{3}{|c|}{$\begin{array}{c}\text { Model } 3 \\
\text { Independent variable } \\
\text { (attitude of beating } \\
\text { children) }\end{array}$} \\
\hline & $\beta$ & $t$ & $p$ & $\beta$ & $t$ & $p$ & $\beta$ & $t$ & $p$ \\
\hline (Constant) & & 25.226 & .000 & & 24.696 & .000 & & 14.356 & .000 \\
\hline Children's age & .057 & 1.741 & .082 & .048 & 1.497 & .135 & .009 & .295 & .768 \\
\hline Children's gender & -.057 & -1.800 & .072 & -.050 & -1.631 & .103 & -.051 & -1.736 & .083 \\
\hline Parents' age & -.118 & -3.622 & .000 & -.109 & -3.437 & .001 & -.118 & -3.932 & .000 \\
\hline Parents' educational background & -.055 & -1.508 & .132 & -.077 & -2.132 & .033 & -.130 & -3.785 & .000 \\
\hline Living region & .125 & 3.513 & .000 & .135 & 3.850 & .000 & .100 & 3.011 & .003 \\
\hline Cognition on anti-domestic violence & & & & -.197 & -6.315 & .000 & -.140 & -4.468 & .000 \\
\hline Purposiveness & & & & & & & .252 & 8.072 & .000 \\
\hline Indirect Blasting Fuse & & & & & & & .180 & 5.896 & .000 \\
\hline Conceptual Factor & & & & & & & .041 & 1.269 & .205 \\
\hline \multicolumn{10}{|l|}{ Model abstract } \\
\hline$R^{2}$ & & .234 & & & .305 & & .446 & & \\
\hline$F$ & & 11.007 & & & 16.195 & & 26.069 & & \\
\hline$p$ & & .000 & & & .000 & & .000 & & \\
\hline$\Delta R^{2}$ & & .055 & & & .038 & & .106 & & \\
\hline$\Delta F$ & & 11.007 & & & 39.885 & & 41.660 & & \\
\hline P of change & & .001 & & & .000 & & .000 & & \\
\hline
\end{tabular}




\section{Discussion}

The analysis results and discussions of this research are as follows:

Firstly, the research has found out that with regard to the frequency of beating children, there are 53 people for Never (5.5\%), 34.4\% of which chose Rarely, 56.4\% of which chose Sometimes, 3.4\% of which chose Usually, and only $0.2 \%$ of which chose Always. This research result is the same as that of Xiao and Chen (2011), whose study showed that $77.7 \%$ of 560 parents have used physical violence on their children within 3 months. That parents use physical violence on children in China, by contrast, is still a common phenomenon. Obviously, parents who have once beaten their child occupy a high rate. The research shows that although there are active ways of education such as setting parents themselves as examples, teaching by personal example, equally communication, patiently guidance, praising and encouragement, etc., there are also negative ways of education such as criticizing, punishment, threatening, etc., even more furious ways like "beating". This common phenomenon of parents beating children should raise more attention in society, and measures should be taken to solve the problem, especially for a small amount of children who are under the situation of usually or always being beaten, aids are urgent for them.

Secondly, with the demographic variable, cognition of anti-domestic violence, and attitude towards beating children as explaining variable, the hierarchical regression analysis have the explanatory power on the frequency of beating children, and there are three analysis results as follows:

Among five demographic variables including children's age, children's gender, parents' age, parents' educational background, and living region, only the factors of parents' age and living region have the significant explanatory power. While the $\beta$ value of parents' age is negative, which means the larger parents' age is, the less the frequency of beating children is. The reason may be that younger parents are more aggressive and easier to get mad, so they couldn't help to beat their children; which may also because that under the Second-child Policy, many parents get their second child in their high age so they tend to dote their child, not choosing beating as a way of education. While the $\beta$ value of the region is positive (city is dummied as 0 , rural area as 1), indicating that the frequency of beating children in cities is lower, which has been found out by Sajkowska (2007) [12]. She found that people in big cities were more opposed to pay corporal punishment for children.

After controlling the explanatory power of demographic variable, it has be found that the cognition of anti-domestic violence has the significant explanatory power, and the $\beta$ value is negative, indicating that the higher cognition of anti-domestic violence is, the lower frequency of beating children is. Laws are constraints on people's behavior, and
China's Anti-Domestic Violence Law (2016)[8] has clear specification of domestic violence that treating the behaviors among family members such as beating, binding, destroying infringement as domestic violence, and also clarifying that guardians mustn't use domestic violence in family education. But after the Anti-Domestic Violence Law has been in effect for more than a year, this study found that the proportion of parents who have beaten their children is high, and there is clearly a lot of room for improvement. Therefore, the government should implement this policy and let parents know the content of this law to reduce the frequency of beating children.

After controlling the demographic variable and the cognition on anti-domestic violence, it has found out that among the three levels of attitude towards beating children, two of them have obvious explanatory power on the frequency of beating children. The explanations of these three levels are as follows:

With regard to purposiveness, researchers have found out that this level has obvious explanatory power, and $\beta$ value is positive, which indicates that the more parents recognize that beating children is for their good, correcting children's behaviors, letting children know what they should do and what they mustn't do, teaching them lessons, or being impatient for children's behaviors, the more possibility they would beat children. Just as Pestalozzi said that "Unless there is no alternative, I will never let my child suffer physical pain" (Lin, 2002) [6] .While the purposiveness of parents beating children is mostly for correcting their behaviors, letting children learn from it, having strong sense of discipline, teaching children to obey orders. Quote Foucault's (1992) [13],opinion in his book Discipline and Punish it can be understood as follows: Parents beat their children with a strong sense of punishment, "punishment is for the future", and by the painful experience of "beating", prevents would like to prevent children from "repeating the same mistakes". Parents spank their children, for example, the specific reason on top of the list found by this study is children's dangerous behavior (based on the data in the questionnaires), suggesting that parents pay more attention to the safety of the children, forbidding children to be engaged in risky behavior by beating. Although this research result is different from that of Cui, Pang, Du, Xue, Ren, Wang and Li (2010) [14], who found that the first reason why parents use physical violence on young children depends on young children's bad living habits. This research has found out that parents spank children because children are engaged in risky behavior or bad living habits. The purpose of parents beating their children has a profound characteristic of expectation for the growth of children, and mostly it is from the point of goodwill, for a better growth of their children. It can be called "deep love and fierce reproach." By beating children, parents realize that "only the love with fear has the effect of education"(Lin Yuti, 2002)[6]. 
With regard to indirect blasting fuse, which refers to parents having a bad mood, parents' not understanding children's physical and mental development characteristics, etc. This research has found out that the $\beta$ value of indirect blasting fuse is positive, indicating that the higher parents' indirect blasting fuse attitude is, the higher frequency of beating children is. The explanatory power of indirect blasting fuse may be derived from depressive pressure from work and life, bad relationship with family members, bad mood at the moment, and other objective factors.

With regard to conception, this level has no explanatory power on the frequency of beating children, which means the conceptions such as "beating children is an effective approach to educate children", "wolf fathers and tiger mothers ${ }^{1}$ being popular on the internet", and "beating the child once every three days, he will be able to be admitted to Peking University" are not the decisive factor of the frequency of beating children. It also shows that the frequency of Chinese parents beating their children has been removed from the traditional culture of what is known as "the child not being beaten couldn't become a talent."

Finally, with regard to the result of hierarchical analysis, the total explanatory value of demographic variable, cognition of anti-domestic violence, attitude towards beating children is $44.60 \%$, which indicates that there are still $55.40 \%$ of total amounts can't be explained by this variable and there are other explanatory variables needed to be explored.

\section{REFERENCES}

[1] L. Y. Zhang. Say goodbye to punishment. Journal of Education Science of Hunan Normal University. No. 2, 55-59, 2013.

[2] Q. Z. Chen. History of China's Education. Commercial Press, Taipei, Taiwan, 1973.

[3] F. Y. Wang, J. Q. Chen, Y, X, Ma. The Prevalence of Physical Maltreatment by Parents in 810 Kindergarten Children. Chinese Journal of School Health. Vol. 28, No. 11, 987-988, 2007.
[4] W. Q. Xiao, J.Q. Chen. Physical violence against children, a survey in 560 kindergarten children's parents /guardians. Chinese Journal of Child Health Care, Vol. 19, No. 3, 230-232, 2011.

[5] J. H. He. The current situation of Chinese children suffering from parental corporal punishment: A study based on The Third Survey on the Social Status of Women in China. Journal of Yunnan Minzu University ( Social Sciences), No. 6, 131-138, 2014.

[6] Y. T. Lin. History of Western Educational Thought (2nd ed.). Sanmin Press, Taipei, Taiwan, 2002.

[7] E.T. Gershoff. Corporal punishment by parents and associated child behaviors and experiences: A meta-analytic and theoretical review. Psychological Bulletin, Vol. 128, No. 4, 539-579, 2002.

[8] Anti-Domestic Violence Law of People's Republic of China (2016)

[9] Y. Guan. Domestic violence's hurt on children and its social intervene. Contemporary Youth Research, Vol.5, No. 1, 22-27, 2006.

[10] J. Sun. My opinion on beating children (question discussion)-It is allowable to beat children properly. Early Education: Teachers' Version, No. 1, 5, 1993.

[11] Y. Y. Gao, L. Ye. Social Cognitive Status, Problems and Recommendations of Children Abuse. China Social Welfare, No. 6, 44-47, 2016.

[12] M. Sajkowska. The Problem of Child Abuse: Attitudes and Experiences in Seven Countries of Central and Eastern Europe. Warsaw University, Warsaw, Poland, 2007.

[13] P. M. Foucault. Discipline and Punish (translated by B. C. Liu \& D. Y. Yang). Guiguan Book, Taipei, Taiwan, 1992.

[14] L. H. Cui, S. L. Pang, W. R. Du, L. Xue, Q. Ren, W. X. Wang, Y. Li. Prevalence of physical and emotional maltreatment by parents in preschool children. Chinese Journal of Public Health, No. 4, 486-487, 2010.

[15] H. S. Yao, S. J. Gao. Sociological Analysis on the phenomenon of "tiger mothers and wolf fathers"-Primary Investigation on family education's view of education model, Journal of Yanan University(Social Science), Vol. 34, No. 5, 123-125, 2012.

\footnotetext{
${ }^{1}$ It means that parents strictly educate children, maybe including the abuse education, and expect their kids could be admitted to a prestigious University. (Yao \& Gao, 2012) [15]
} 